\title{
Improvement of Mechanical Welding Properties by using Induced Harmonic Vibration.
}

\begin{abstract}
The enhancement of the welding mechanical properties and the quality of the fusion metal is considered recently by using vibration during welding. In this study, the effect of induced harmonic vibration during welding is employed to improve the welding mechanical properties and to reach the best shape of welding line on the surface. The harmonic vibration method is examined experimentally by using four values of mechanical frequency during welding on the ductility, tensile strength and the homogeneity of the welding line. The frequencies were specified according to the natural frequency of the plate. Five simply supported rectangular plates are supported on the supporting stand and welded using a manual arc-welding machine. The experimental results show that the vibration applied during welding generally improved the bend property of the welding line, as well as the tensile strength has been improved distinctively at the resonance case when compared with that one welded without vibration. The morphology of the fillet metal after welding and for each value of frequency show an enhancement in the distribution of the fusion fillet metal, with gradually disappearing of the micro crack that may shown inside the metal with increasing the mechanical frequency. A comparison between the properties of welding without vibration and welding with vibration is discussed.
\end{abstract}

Keyword: Welding; Vibration 